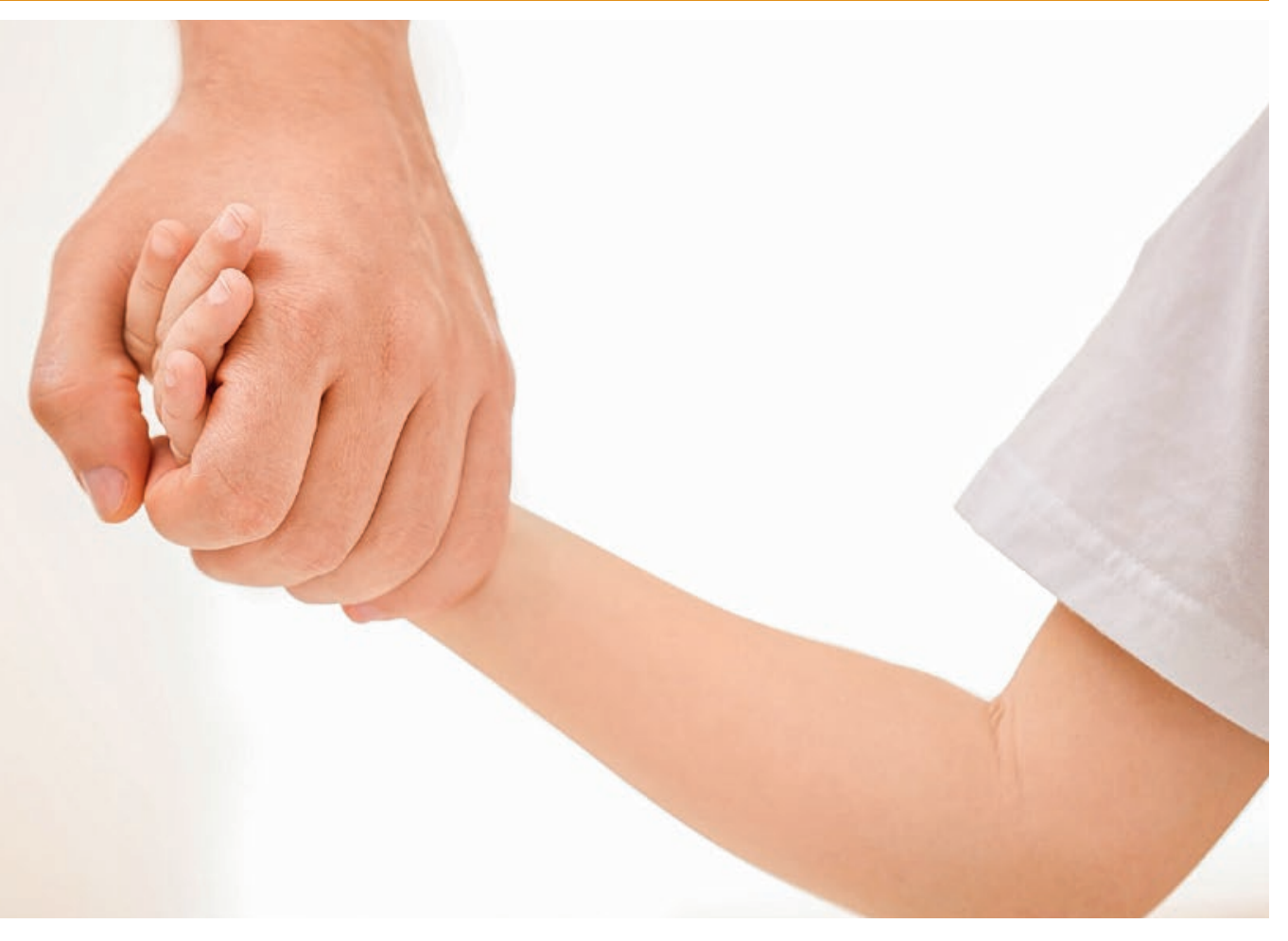

\title{
Betreuungsurlaub für Eltern schwer kranker Kinder
}

\section{Andrea Künzli}

MLaw, Rechtsanwältin, Bundesamt für Sozialversicherungen (BSV), Bern

Eltern erhalten ab Juli 2021 einen 14-wöchigen Betreuungsurlaub, wenn sie ihr gesundheitlich schwer beeinträchtigtes Kind betreuen und deswegen ihre Erwerbstätigkeit unterbrechen müssen. Die gesundheitliche Beeinträchtigung des Kindes ist ärztlich zu bescheinigen. Die Leitplanken, wann eine schwere gesundheitliche Beeinträchtigung vorliegt, gibt das Erwerbsersatzgesetz (EOG) vor.

Das Parlament hat die Bedingungen für betreuende und pflegende Angehörige mit konkreten Massnahmen verbessert [1]. Sie sind in einem Mantelerlass zusammengefasst [2]. Eine dieser Massnahmen ist der Betreuungsurlaub für Eltern gesundheitlich schwer beeinträchtigter Kinder (Art. 329i OR), der auf den 1. Juli 2021 umgesetzt wird.

\section{Eckwerte}

Der Betreuungsurlaub dauert maximal 14 Wochen und ist innerhalb einer Rahmenfrist von 18 Monaten am Stück, tage- oder wochenweise zu beziehen. Betroffene Eltern können ihre Erwerbstätigkeit zur Betreuung ihres gesundheitlich schwer beeinträchtigten Kindes unterbrechen und haben in dieser Zeit Anspruch auf eine Betreuungsentschädigung. Die Betreuungsentschädigung ist Eltern vorbehalten, deren minderjähriges Kind wegen der schweren gesundheitlichen Beeinträchtigung einen erhöhten Bedarf an Betreuung hat. Anspruch auf den Betreuungsurlaub haben neben Arbeitnehmenden oder Selbständigerwerbenden unter bestimmten Bedingungen auch arbeitslose oder arbeitsunfähige Eltern.

\section{Gesundheitliche Beeinträchtigung}

Die Ärztin respektive der Arzt bescheinigt direkt im Anmeldeformular für die Betreuungsentschädigung, dass das Kind gesundheitlich schwer beeinträchtigt im 
Sinne des Gesetzes ist. Mit Kindern sind Minderjährige gemeint, wobei der Anspruch auf den Betreuungsurlaub aber nicht vorzeitig endet, wenn das Kind während der 18-monatigen Rahmenfrist volljährig wird. Das Anmeldeformular können die Eltern bei der zuständigen AHV-Ausgleichskasse oder über www. ahv-iv.ch beziehen. Die AHV-Ausgleichskasse, bei der die Betreuungsentschädigung zu beantragen ist, ist an die ärztliche Bescheinigung gebunden.

Das Gesetz enthält bewusst keine Definition der schweren gesundheitlichen Beeinträchtigung. Vielmehr soll möglichst die gesamte Bandbreite schwerer gesundheitlicher Beeinträchtigungen umfasst werden und gleichzeitig eine Abgrenzung zur mittelschweren Beeinträchtigung erfolgen. Als mittelschwere Beeinträchtigungen gelten solche, die zwar Spitalaufenthalte oder regelmässige Arztbesuche erforderlich machen und den Alltag erschweren, bei denen aber mit einem positiven Ausgang zu rechnen oder die gesundheitliche Beeinträchtigung kontrollierbar ist (z.B. Knochenbrüche, Diabetes, Lungenentzündung). Gemäss Artikel 16o Buchstabe a bis d EOG ist ein Kind gesundheitlich schwer beeinträchtigt, wenn (kumulativ):

a. eine einschneidende Veränderung seines körperlichen oder psychischen Zustandes eingetreten ist.

Massgebend für die Beurteilung des Schweregrads der gesundheitlichen Beeinträchtigung des Kindes sind zunächst die Symptome der gesundheitlichen Beeinträchtigung. Diese muss eine stationäre oder ambulante ärztliche Behandlung des Kindes über eine längere Dauer (mehrere Monate) bedingen, wobei die Dauer zu Beginn häufig noch nicht abschätzbar ist. Das Gesetz sieht keine Minimaldauer der Behandlung vor.

\section{Die Eltern können den Betreuungsurlaub frei unter sich aufteilen.}

Damit ist in erster Linie der Eintritt einer akuten Krankheitssituation gemeint. Aber auch die schleichende Verschlimmerung des Gesundheitszustandes, die ab einer bestimmten Intensität eine Betreuung erfordert, oder die akute Verschlechterung des Gesundheitszustandes bei einem chronisch kranken Kind.

b. der Verlauf oder der Ausgang dieser Veränderung schwer vorhersehbar ist oder mit einer bleibenden oder zunehmenden Beeinträchtigung oder dem Tod zu rechnen ist.

Ist der Verlauf ungewiss und schwer vorhersehbar, kann das bedeuten, dass der Heilungsverlauf mit Schwankungen einhergeht, mit Rückfällen zu rechnen ist und auch der Ausgang des Heilungsverlaufs offen ist. Mit dem Kriterium der schweren Vorher- sehbarkeit geht die Vermutung einher, dass sich der Verlauf über eine längere Dauer hinziehen wird; eine Mindestdauer wurde aber bewusst nicht festgelegt.

c. ein erhöhter Bedarf an Betreuung durch die Eltern besteht.

Schwere gesundheitliche Beeinträchtigungen verlangen eine intensive Betreuung durch die Eltern. Das Ausmass des Betreuungsbedarfs ist neben der Schwere der gesundheitlichen Beeinträchtigung wesentlich vom Alter des Kindes abhängig. Insofern kann dieselbe gesundheitliche Beeinträchtigung je nach Alter des Kindes als schwer oder weniger schwer beurteilt werden.

\section{Das Gesetz enthält bewusst keine Definition der schweren gesundheitlichen Beeinträch- tigung.}

Mit Betreuung ist auch Beistehen bei Besprechungen, Arzt- oder Spitalbesuchen gemeint. Indes ist ein schwerkrankes Kind unter Umständen nicht dauernd auf Betreuung angewiesen. Es kann Phasen geben, in denen es den Alltag selbständig meistert, und andere, in denen es auf Hilfe angewiesen ist. Dies kann insbesondere auf psychisch Kranke zutreffen. Die enge Betreuung soll auch Phasen einschliessen, in denen sich die Betreuung auf das eigentliche Beistehen beschränkt (z.B. während eines langen Spitalaufenthalts) und konkrete Pflegeund Betreuungshandlungen nur von Fachpersonen vorgenommen werden können.

d. mindestens ein Elternteil die Erwerbstätigkeit für die Betreuung des Kindes unterbrechen muss.

Die Begleitung, Betreuung oder Pflege muss so aufwendig sein, dass die Erwerbstätigkeit von mindestens einem Elternteil unterbrochen werden muss. Dabei ist aber keine Mindestanzahl an bestimmten Betreuungshandlungen oder Betreuungsstunden pro Tag erforderlich.

\section{Aufteilung unter den Eltern}

Die Eltern können den Betreuungsurlaub frei unter sich aufteilen. Wenn sich die Eltern nicht über die Aufteilung einigen können, hat jeder Elternteil Anspruch auf sieben Wochen Betreuungsurlaub. Den Eltern steht es frei, den Urlaub gleichzeitig zu beziehen.

\section{Rahmenfrist}

Die Rahmenfrist von 18 Monaten beginnt an dem Tag zu laufen, für den der erste der beiden Elternteile eine Betreuungsentschädigung bezieht. Die Rahmenfrist 
ist an das Kind gebunden und verschiebt sich deshalb nicht, wenn ein Elternteil später als der oder die Erstbeziehende eine Betreuungsentschädigung beansprucht. Bei mehreren anspruchsbegründenden Kindern löst jedes einzelne Kind eine Rahmenfrist aus. Erleidet das Kind einen Rückfall oder tritt ein neues Ereignis ein, beginnt eine neue Rahmenfrist zu laufen.

\section{Berechnung und Höhe}

Für die Berechnung der Betreuungsentschädigung ist das durchschnittliche Einkommen massgebend, das der jeweilige Elternteil unmittelbar vor dem Bezug der entsprechenden Urlaubstage erzielt hat. Die Entschädigung beläuft sich auf 80 Prozent dieses Einkommens, höchstens aber auf 196 Franken pro Tag. Die Einkommen der Eltern werden für die Berechnung der Betreuungsentschädigung nicht addiert; auch die Auszahlung erfolgt gesondert.

\section{Anmeldung und Durchführung}

Der Anspruch auf die Betreuungsentschädigung ist bei der zuständigen AHV-Ausgleichskasse geltend zu machen. Diese prüft, ob die Anspruchsvoraussetzungen erfüllt sind. Dabei ist sie an die ärztliche Bescheinigung, die Bestandteil der Anmeldung ist, gebunden. Sie muss also nicht prüfen, ob die medizinischen Voraussetzungen erfüllt sind. Indem der Arbeitgeber die Betreuungsentschädigung bei der zuständigen AHVAusgleichskasse beantragt, bestätigt er die Glaubwürdigkeit der ärztlichen Bescheinigung.

Nach erfolgter Anmeldung reichen der Arbeitgeber oder die Duchführungsorgane der Arbeitslosenversicherung der zuständigen Ausgleichskasse monatlich eine Bescheinigung über die im vergangenen Monat bezogenen Urlaubstage ein. Gestützt auf diese Meldungen berechnet die AHV-Ausgleichskasse die Be- treuungsentschädigung und prüft, wie viele Tage Betreuungsurlaub noch bezogen werden können. Diese Informationen teilt sie den Eltern und dem Arbeitgeber oder den zuständigen Durchführungsorganen der Arbeitslosenversicherung mit.

Der Betreuungsurlaub entlastet betroffene Eltern. Sie können sich um ihre kranken Kinder kümmern, ohne den Verlust der Arbeitsstelle oder eine empfindliche

\section{Die Entschädigung beläuft sich auf 80 Pro-} zent dieses Einkommens, höchstens aber auf 196 Franken pro Tag.

Erwerbseinbusse befürchten zu müssen. Die Lage betroffener Familien verbessert sich somit ab Juli 2021 deutlich, auch wenn der Urlaub nicht in allen Fällen die ganze Zeit abdecken kann, die zur Betreuung notwendig wäre.

\section{Bildnachweis}

Chernetskaya | Dreamstime.com

\section{Literatur}

1 Bundesrat (2019): Botschaft vom 22. Mai 2019 zum Bundesgesetz über die Verbesserung der Vereinbarkeit von Erwerbstätigkeit und Angehörigenbetreuung (19.027), in BBl 2019 4103: www.admin.ch $\rightarrow$ Bundesrecht $\rightarrow$ Bundesblatt $\rightarrow 2019$.

2 AS 2020 4525, Bundesgesetz über die Verbesserung der Vereinbarkeit von Erwerbstätigkeit und Angehörigenbetreuung vom 20. Dezember 2019.

\section{Das Wichtigste in Kürze}

- $\quad$ Ab dem 1. Juli 2021 haben Eltern Anspruch auf einen 14-wöchigen Betreuungsurlaub, wenn sie ihr gesundheitlich schwer beeinträchtigtes Kind betreuen und deswegen ihre Erwerbstätigkeit unterbrechen müssen.

- Die gesundheitliche Beeinträchtigung des Kindes muss dabei ärztlich bescheinigt werden.

- Wann ein Kind als gesundheitlich schwer beeinträchtigt gilt, gibt das Erwerbsersatzgesetz (EOG) vor. 